\title{
Hereditary Convexity for Harmonic Homeomorphisms
}

\author{
NGIN-TEE KOH
}

\begin{abstract}
We study hereditary properties of convexity for planar harmonic homeomorphisms on a disk and an annulus. A noteworthy class of examples with the hereditary property arises from energy-minimal diffeomorphisms of an annulus, whose existence was established in $[9,11]$. An extension of a result by Hengartner and Schober [8] to an annulus is used to deduce the boundary behavior of a harmonic mapping from an annulus into a doubly-connected region bounded by two convex Jordan curves.
\end{abstract}

\section{INTRODUCTION}

Harmonic mappings, which are complex-valued orientation-preserving univalent functions satisfying Laplace's equation $\Delta f=0$ on their respective domains in $\mathbb{C}$, bear some curious features. For example, while harmonic mappings of hyperbolic regions generally do not decrease either the Euclidean metric or the hyperbolic metric (because a result of Heinz [6, Lemma] is optimal—see, e.g., [4, p. 77] or $[12$, p. 91]), it was shown in [12, Theorem 1.1] that harmonic mappings preserving the unit disk $\mathbb{D}$ decrease the Lebesgue area measure of concentric disks $\mathbb{D}_{r}=\{z \in \mathbb{C}:|z| \leq r<1\}$.

If the image of the unit disk under a conformal mapping is a convex region $\Omega$, then the image of every disk in $\mathbb{D}$ is also convex (see, e.g., [3, proof of Theorem 2.11] or [16]). On the other hand, the situation for harmonic mappings is markedly different. The harmonic mapping

$$
f(z)=\operatorname{Re} \frac{z}{1-z}+i \operatorname{Im} \frac{z}{(1-z)^{2}}
$$


maps $\mathbb{D}$ onto the half plane $\left\{w: w>-\frac{1}{2}\right\}$, which is convex, but $f\left(\mathbb{D}_{r}\right)$ is not convex for $\sqrt{2}-1<r<1$ (see, e.g., [2, Example 5.5] or [4, pp. 46-48]). This is related to the fact that unidirectional convexity is not a hereditary property of holomorphic univalent functions (see, e.g., [2, Theorem 5.3; 5; 7]). Hence, convexity is not a hereditary property of harmonic mappings in general. Nevertheless, we obtain sufficient conditions for this hereditary property to be present in harmonic mappings. We also study a related hereditary property of harmonic mappings between doubly-connected regions, which is the main subject of this paper.

\section{Connection with the Doubly-Connected Case}

For $0<r<1$, let $\mathbb{A}_{r}$ denote the annulus $\{z \in \mathbb{C}: r<|z|<1\}$, let $\overline{\mathbb{A}_{r}}$ denote its closure, and let $\mathbb{T}_{r}$ denote the circle $\{z \in \mathbb{C}:|z|=r\}$. We will use $\mathbb{T}$ to represent the unit circle $\partial \mathbb{D}$. Harmonic diffeomorphisms will refer to harmonic mappings that are diffeomorphisms.

At first glance, our following result may appear somewhat surprising.

Theorem 2.1. Let $h$ be a harmonic diffeomorphism of $\mathbb{D}$ into a bounded convex region $\Omega_{0}$ in the finite plane such that the radial limit $\lim _{r \rightarrow 1} h\left(r e^{i \theta}\right)$ lies on $\partial \Omega_{0}$ for almost all $\theta$. Suppose that, on $\mathbb{A}_{\sqrt{2}-1}$,

$$
\Delta \operatorname{Im} \log \left(1-\frac{\bar{z} h_{\bar{z}}}{z h_{z}}\right)=0,
$$

where $h_{z}=\partial h / \partial z, h_{\bar{z}}=\partial h / \partial \bar{z}$, and $\Delta$ represents the Laplace operator. Then, $h\left(\mathbb{D}_{r}\right)$ is a strictly convex region for $0<r<1$.

Remark. If $h$ is conformal, then $1-\bar{z} h_{\bar{z}} /\left(z h_{z}\right) \equiv 1$. Hence, its argument function $\operatorname{Im} \log \left(1-\bar{z} h_{\bar{z}} /\left(z h_{z}\right)\right)$ is a constant integer multiple of $2 \pi$.

In view of condition (2.1), we have stated Theorem 2.1 for harmonic diffeomorphisms in place of harmonic mappings. This is nonetheless hardly a restriction, since a result of Lewy (see, e.g., [4, p. 20] or [14, Theorem 1]) shows that the Jacobian of a harmonic mapping does not vanish at any point.

A harmonic mapping $f$ of $\mathbb{D}$ into a bounded region has bounded real and imaginary parts. By Fatou's Theorem [17, Theorem IV.6], the angular limits of $f$ exist almost everywhere on $\partial \mathbb{D}$. Hence, the radial limit assumption in Theorem 2.1 is apparently weaker than customarily requiring either the convexity of $h(\mathbb{D})$ or the surjectivity of $h$.

Given a harmonic mapping $g$ of $\mathbb{D}$, where $g(\mathbb{D})$ is a convex region, it is known that $g\left(\mathbb{D}_{r}\right)$ is convex for $r \in(0, \sqrt{2}-1$ ] (see, e.g., [4, p. 46] or [15, Theorem 1]). This explains the focus on $\mathbb{A}_{\sqrt{2}-1}$ in Theorem 2.1. More generally, we prove the following.

Theorem 2.2. Let $h$ be a harmonic diffeomorphism of $\mathbb{A}_{\rho}$ into a doubly-connected region $\Omega$ bounded by two convex Jordan curves in the finite plane such that the 
radial limits $\lim _{r \rightarrow 1} h\left(r e^{i \theta}\right)$ and $\lim _{r \rightarrow \rho} h\left(r e^{i \varphi}\right)$ lie on $\partial \Omega$ for almost all $\theta$ and $\varphi$. If $(2.1)$ holds on $\mathbb{A}_{\rho}$, then $h\left(\mathbb{T}_{r}\right)$ is a strictly convex curve for $\rho<r<1$.

Suppose $f$ is a bounded harmonic mapping of $\mathbb{A}_{\rho}$. The compact set $\partial \mathbb{A}_{\rho}$ may be covered by a finite number of simply-connected neighborhoods $R_{1}, R_{2}$, $\ldots, R_{n}$ in $\overline{\mathbb{A}_{\rho}}$ whose boundaries are Jordan curves. For each integer $k \in[1, n]$, let $g_{k}$ be a conformal mapping of $\mathbb{D}$ onto the interior of $R_{k}$. By Fatou's Theorem, the harmonic mapping $f \circ g_{k}$ has angular limits almost everywhere on $\mathbb{T}$. The isogonality of $g_{k}$ at each boundary point of $\mathbb{T}$ (see, e.g., [17, Theorem IX.5 and the subsequent paragraph]) implies that $f$ has angular limits almost everywhere on $\partial \mathbb{A}_{\rho} \cap R_{k}$. It follows that $f$ has angular limits almost everywhere on $\partial \mathbb{A}_{\rho}=\bigcup_{k=1}^{n}\left(\partial \mathbb{A}_{\rho} \cap R_{k}\right)$. Hence, the radial limit assumption in Theorem 2.2 may appear to be weaker than requiring either the boundary components of $h\left(\mathbb{A}_{\rho}\right)$ to be convex Jordan curves or $h$ to be surjective. While the latter comparison is correct, it will follow from Corollary 4.2 in Section 4 that the radial limit assumption in Theorem 2.2 implies that the boundary components of $h\left(\mathbb{A}_{\rho}\right)$ are convex Jordan curves.

\section{Illustrative ExAmples}

Definition 3.1. An orientation-preserving homeomorphism $h: \mathbb{A}_{\rho} \rightarrow \Omega$ is said to be energy-minimal if $h$ minimizes the quantity

$$
E(f)=\iint_{\mathbb{A}_{\rho}}\left|f_{z}\right|^{2}+\left|f_{\bar{z}}\right|^{2}
$$

among all orientation-preserving homeomorphisms $f: \mathbb{A}_{\rho} \rightarrow \Omega$ with $E(f)<\infty$.

Remark 3.2. An energy-minimal homeomorphism $h: \mathbb{A}_{\rho} \longrightarrow \Omega$ exists as long as the conformal modulus of $\mathbb{A}_{\rho}$ does not exceed that of $\Omega$ (see, e.g., [9, Theorem 1.1] or [11, Theorem 1.1]).

Remark 3.3. Energy-minimal homeomorphisms are diffeomorphisms [10, Theorem 1.2]. This can also be seen as a consequence of their harmonicity (see, e.g., [14, Theorem 1] and the next remark). Henceforth, we refer to them as energy-minimal diffeomorphisms.

Remark 3.4. Since Poisson modification decreases the quantity in (3.1) (see, e.g., [1, proof of Lemma 7] or [9, Lemma 4.2]), energy-minimal diffeomorphisms are necessarily harmonic [9, Proposition 8.1 and Theorem 2.3].

It turns out that an energy-minimal diffeomorphism $h: \mathbb{A}_{\rho} \rightarrow \Omega$ satisfies (2.1). Since $h$ is an orientation-preserving diffeomorphism,

$$
\left|h_{z}\right|>\left|h_{\bar{z}}\right|
$$

on $\mathbb{A} \rho$. It was shown in $[9$, Lemma 6.1] that

$$
h_{z} \overline{h_{\bar{z}}}=\frac{m}{z^{2}},
$$


where $m$ is a real constant. We can rewrite (3.3) as

$$
\frac{\bar{z} h_{\bar{z}}}{z h_{z}}=\frac{m}{|z|^{2}\left|h_{z}\right|^{2}},
$$

which, in view of (3.2), yields

$$
-1<\frac{\bar{z} h_{\bar{z}}}{z h_{z}}<1,
$$

and thus the function $1-\bar{z} h_{\bar{z}} /\left(z h_{z}\right)$ is real and positive. Consequently, its argument function $\operatorname{Im} \log \left(1-\bar{z} h_{\bar{z}} /\left(z h_{z}\right)\right)$ is a constant integer multiple of $2 \pi$. Theorem 2.2 now yields the following result.

Theorem 3.5. Let $h: \mathbb{A}_{\rho} \rightarrow \Omega$ be an energy-minimal diffeomorphism, where $\Omega$ is a doubly-connected region bounded by two convex Jordan curves in the finite plane. Then, $h\left(\mathbb{T}_{r}\right)$ is a strictly convex curve for $\rho<r<1$.

We conclude this section with a family of examples for which

$$
\operatorname{Im} \log \left(1-\frac{\bar{z} h_{\bar{z}}}{z h_{z}}\right)
$$

is not constant. Define $h: \mathbb{A}_{\rho} \rightarrow \mathbb{C}$ by

$$
h(z)=\frac{z+a}{1+a z}-b \log |z|,
$$

where $a \in(0,1)$ and $b=a\left(1-\rho^{2}\right) /\left(\left(1-\rho^{2} a^{2}\right) \log \rho\right)<0$. Then, $h$ is harmonic, and

$$
1-\frac{\bar{z} h_{\bar{z}}}{z h_{z}}=\frac{2\left(1-a^{2}\right) z}{2\left(1-a^{2}\right) z-b(1+a z)^{2}},
$$

which is meromorphic on $\mathbb{A}_{\rho}$. Another elementary computation shows that the Jacobian

$$
\left|h_{z}\right|^{2}-\left|h_{\bar{z}}\right|^{2}=\frac{\left(1-a^{2}\right)^{2}}{|1+a z|^{4}}\left(1-\frac{b}{1-a^{2}} \operatorname{Re} \frac{(1+a z)^{2}}{z}\right),
$$

whose last factor on the right-hand side has its minimum on $\overline{\mathbb{A}_{\rho}}$ at $z=-\rho$. Hence, $h$ will be a harmonic diffeomorphism of $\mathbb{A}_{\rho}$ onto $\mathbb{A}_{\sigma}$ satisfying (2.1) for values of $a$ and $\rho$ such that this minimum is positive, where we have that $\sigma=\rho\left(1-a^{2}\right) /\left(1-\rho^{2} a^{2}\right) \in(0, \rho)$. An instance of this occurs at $a=\rho=\frac{1}{2}$.

\section{Auxiliary Results on Boundary Behavior}

Let $\theta$ and $\varphi$ denote polar angles. In this section, we study the boundary behavior of a harmonic mapping between an annulus $\mathbb{A}_{\rho}$ and a doubly-connected region $\Omega$ bounded by two Jordan curves that is not necessarily surjective, but whose radial limits at $\partial \mathbb{A}_{\rho}$ are contained in $\partial \Omega$. 
Proposition 4.1. Let $h$ be a harmonic mapping of $\mathbb{A}_{\rho}$ into a doubly-connected region $\Omega$ bounded by two Jordan curves $C_{1}$ and $C_{\rho}$ in the finite plane such that the radial limits $\lim _{r \rightarrow 1} h\left(r e^{i \theta}\right)$ and $\lim _{r \rightarrow \rho} h\left(r e^{i \varphi}\right)$ lie on $C_{1}$ and $C_{\rho}$, respectively, for almost all $\theta$ and $\varphi$. Then, there is a countable set $W \subset \partial \mathbb{A}_{\rho}=\mathbb{T} \cup \mathbb{T} \rho$ such that the unrestricted limits

$$
H\left(e^{i \theta}\right)=\lim _{z \rightarrow e^{i \theta}} h(z), \quad H\left(\rho e^{i \varphi}\right)=\lim _{z \rightarrow \rho e^{i \varphi}} h(z)
$$

exist on $\partial \mathbb{A}_{\rho} \backslash W$, and are contained in $C_{1}$ and $C_{\rho}$, respectively. Moreover, we have the following:

(a) $H$ is both continuous and orientation-preserving on $\mathbb{T} \backslash W$ and $\mathbb{T}_{\rho} \backslash W$.

(b) For each $e^{i \theta} \in W$, the one-sided limits

$$
\begin{aligned}
& H\left(e^{i \theta-}\right)=\lim _{\sigma \rightarrow \theta^{-}, e^{i \sigma} \notin W} H\left(e^{i \sigma}\right), \\
& H\left(e^{i \theta+}\right)=\lim _{\sigma \rightarrow \theta^{+}, e^{i \sigma} \notin W} H\left(e^{i \sigma}\right)
\end{aligned}
$$

exist, belong to $C_{1}$ and are distinct.

(c) For each $\rho e^{i \varphi} \in W$, the one-sided limits

$$
\begin{aligned}
& H\left(\rho e^{i \varphi-}\right)=\lim _{\sigma \rightarrow \varphi^{-}, \rho e^{i \sigma \notin W}} H\left(\rho e^{i \sigma}\right), \\
& H\left(\rho e^{i \varphi+}\right)=\lim _{\sigma \rightarrow \varphi^{+}, \rho e^{i \sigma} \notin W} H\left(\rho e^{i \sigma}\right)
\end{aligned}
$$

exist, belong to $C_{\rho}$ and are distinct.

(d) The cluster sets of $h$ at the points $e^{i \theta} \in W$ and $\rho e^{i \varphi} \in W$ are the line segments joining $H\left(e^{i \theta-}\right)$ to $H\left(e^{i \theta+}\right)$ and $H\left(\rho e^{i \varphi-}\right)$ to $H\left(\rho e^{i \varphi+}\right)$, respectively.

A version of the above result for harmonic mappings between $\mathbb{D}$ and bounded simply-connected regions with locally-connected boundary was given by Hengartner and Schober [8, Theorem 4.3]. Since the conclusions concern local properties of $h$, we may obtain Proposition 4.1 by covering $\partial \mathbb{A}_{\rho}$ with a finite number of simply-connected neighborhoods $R_{1}, R_{2}, \ldots, R_{n}$ in $\overline{\mathbb{A}_{\rho}}$ whose boundaries are Jordan curves, and applying Hengartner and Schober's result to the harmonic mapping $h \circ g_{k}$, where $g_{k}$ is a conformal mapping of $\mathbb{D}$ onto the interior of $R_{k}$ for each integer $k \in[1, n]$. A noteworthy consequence of Proposition 4.1, besides Corollary 4.2 below, is that $h$ may be extended continuously to $\partial \mathbb{A}_{\rho}$ outside the countable set $W$, and that each boundary point of $h\left(\mathbb{A}_{\rho}\right)$ corresponds to a nonempty "pre-image" (with infinitely many points on a boundary line segment of $h\left(\mathbb{A}_{\rho}\right)$ associated with a "pre-image" point in $W$ from (d)). These facts will prove their worth in Section 5. 
Suppose $f$ is a harmonic mapping of $\mathbb{A}_{\rho}$ into a doubly-connected region $\Omega$ bounded by two convex Jordan curves in the finite plane such that the radial limits $\lim _{r \rightarrow 1} f\left(r e^{i \theta}\right)$ and $\lim _{r \rightarrow \rho} f\left(r e^{i \varphi}\right)$ lie on $\partial \Omega$ for almost all $\theta$ and $\varphi$. The radial limits $\lim _{r \rightarrow 1} f\left(r e^{i \theta}\right)$ and $\lim _{r \rightarrow \rho} f\left(r e^{i \varphi}\right)$ are contained in distinct boundary components of $f\left(\mathbb{A}_{\rho}\right)$ by virtue of the fact that $f$ is a homeomorphism (see, e.g., $[13$, p. 11]), and thus they lie on distinct boundary components of $\partial \Omega$. It follows from Proposition 4.1 that the boundary of $f\left(\mathbb{A}_{\rho}\right)$ consists of two Jordan curves. Since any inner boundary line segment of $f\left(\mathbb{A}_{\rho}\right)$ has to be a subset of the inner boundary of $\Omega$, the inner boundaries of $f\left(\mathbb{A}_{\rho}\right)$ and $\Omega$ must coincide. On the other hand, replacing any outer boundary sub-arc $\gamma$ of $\partial \Omega$ with a line segment joining the endpoints of $\gamma$ results in a convex Jordan curve. Hence, we have the following result.

Corollary 4.2. Let $h$ be a harmonic mapping of $\mathbb{A}_{\rho}$ into a doubly-connected region $\Omega$ bounded by two convex Jordan curves in the finite plane such that the radial limits $\lim _{r \rightarrow 1} h\left(r e^{i \theta}\right)$ and $\lim _{r \rightarrow \rho} h\left(r e^{i \varphi}\right)$ lie on $\partial \Omega$ for almost all $\theta$ and $\varphi$. Then, the boundary of $h\left(\mathbb{A}_{\rho}\right)$ consists of two convex Jordan curves, of which the inner boundary curve coincides with the inner boundary curve of $\Omega$.

\section{SECANT BEHAVIOR NEAR THE Boundary}

Suppose $h$ is a harmonic diffeomorphism of $\mathbb{A}_{\rho}$ into a doubly-connected region $\Omega$ bounded by two convex Jordan curves in the finite plane such that the radial limits $\lim _{r \rightarrow 1} h\left(r e^{i \theta}\right)$ and $\lim _{r \rightarrow \rho} h\left(r e^{i \varphi}\right)$ lie on $\partial \Omega$ for almost all $\theta$ and $\varphi$. For each $\tau>0$, let

$$
f_{\tau}\left(r e^{i \theta}\right)=\frac{h\left(r e^{i(\theta+\tau)}\right)-h\left(r e^{i \theta}\right)}{\tau},
$$

and let $\psi_{\tau}(z)=\arg f_{\tau}(z)$ for all $z=r e^{i \theta} \in \mathbb{A}_{\rho}$. We will establish the following result.

Lemma 5.1. The period of $\psi_{\tau}-\theta$ is $2 \pi$, and the single-valued harmonic functions $\psi_{\tau}-\theta$ are uniformly bounded on $\mathbb{A}_{\rho}$ for sufficiently small $\tau$.

Since the radial $\operatorname{limits}_{\lim _{r \rightarrow 1}} h\left(r e^{i \theta}\right)$ and $\lim _{r \rightarrow \rho} h\left(r e^{i \varphi}\right)$ are contained in distinct boundary components of $\partial \Omega$, it follows from Proposition 4.1 that $h$ has a continuous extension to $\overline{\mathbb{A}_{\rho}} \backslash W$ for some countable set $W \subset \partial \mathbb{A}_{\rho}$. The orientationpreserving feature of $h$ carries over to $\mathbb{T} \backslash W$ and $\mathbb{T}_{\rho} \backslash W$, and by Corollary 4.2, the boundary components of $h\left(\mathbb{A}_{\rho}\right)$ are convex Jordan curves, one of which is a curve $\Gamma$ containing a point $a^{\prime}$ such that

$$
\left|a^{\prime}\right|=\sup _{z \in \mathbb{A}_{\rho}}|h(z)| .
$$

We may suppose, without loss of generality, that

$$
h(\mathbb{T} \backslash W) \subseteq \Gamma .
$$


The line $L$ through $a^{\prime}$ that makes an angle of $\arg a^{\prime}+\pi / 2$ with the positive real axis is a supporting line of $\Gamma$. We let $c^{\prime}$ be a point on $\Gamma$ that has a parallel supporting line distinct from $L$, and pick distinct points $b^{\prime}$ and $d^{\prime}$ on $\Gamma$ that have supporting lines parallel to the line segment $a^{\prime} c^{\prime}$. The points $a^{\prime}, b^{\prime}, c^{\prime}$, and $d^{\prime}$ are chosen so that they follow one another in the positive direction around $\Gamma$, and we denote the angles made by their supporting lines with the positive real-axis by $\alpha$, $\beta, \alpha+\pi$, and $\beta+\pi$, respectively, such that

$$
\alpha<\beta<\alpha+\pi<\beta+\pi<\alpha+2 \pi .
$$

By virtue of the results obtained in Section 4 (see, e.g., Proposition 4.1 and the subsequent paragraph), we may choose on $\mathbb{T}$ four associated "pre-image" points $a, b, c$, and $d$ of $a^{\prime}, b^{\prime}, c^{\prime}$, and $d^{\prime}$, respectively. Let $A, B, C$, and $D$ be the overlapping open arcs on $\mathbb{T}$ from $a$ to $c$, from $b$ to $d$, from $c$ to $a$, and from $d$ to $b$, respectively. We then cover $\mathbb{T}$ with a finite number of sufficiently small open disks $D_{1}, D_{2}, \ldots, D_{n}$ such that the following hold:

(1) Each disk $D_{k}$ intersects $\mathbb{T}$ in an arc $A_{k}$ that is contained within at least one of the arcs $A, B, C$, or $D$.

(2) The endpoints $a_{k}, b_{k}$ of each $A_{k}$ do not coincide with any of the points $a, b, c$, or $d$.

By so doing, we obtain a finite number of simply-connected sets

$$
R_{1}=\overline{\mathbb{A}_{\rho}} \cap D_{1}, R_{2}=\overline{\mathbb{A}_{\rho}} \cap D_{2}, \ldots, R_{n}=\overline{\mathbb{A}_{\rho}} \cap D_{n}
$$

in $\overline{\mathbb{A}_{\rho}}$ whose respective boundaries are Jordan curves, and $R_{k} \cap \mathbb{T}=A_{k}$ for each $k$. Fix $\delta \in(0, \pi / 2)$. For each integer $k \in[1, n]$, let $g_{k}$ be a conformal mapping of $\mathbb{D}$ onto the interior $R_{k}^{\circ}$ of $R_{k}$ such that the harmonic measure

$$
\omega\left(g_{k}(0), A_{k}, R_{k}^{\circ}\right)=2 \delta \text { and } A_{k}=\left\{g_{k}\left(e^{i s}\right):-\delta<s<\delta\right\} .
$$

This may be achieved by extending $g_{k}$ to a homeomorphism of $\overline{\mathbb{D}}$ and making appropriate choices of $g_{k}(0)$ and $g_{k}\left(e^{i s}\right)$ for one particular $s$. If

$$
J=\{s:-\pi<s<-2 \delta\} \cup\{s: 2 \delta<s \leq \pi\},
$$

then for all $s \in J$ and $t_{k} \in(-\delta, \delta)$, we have

$$
\delta<\left|s-t_{k}\right|<\frac{3 \pi}{2}, \quad \cos \left(s-t_{k}\right)<\cos \delta,
$$

and thus

$$
\begin{aligned}
1-2 r_{k} \cos (s-t)+r_{k}^{2} & >1-2 r_{k} \cos \delta+r_{k}^{2} \\
& =\sin ^{2} \delta+\left(r_{k}-\cos \delta\right)^{2} \geq \sin ^{2} \delta .
\end{aligned}
$$


We fix

$$
\tau \in\left(0, d\left(\{a, b, c, d\}, \bigcup_{k=1}^{n}\left\{a_{k}, b_{k}\right\}\right)\right),
$$

where $d(X, Y)=\inf \{d(x, y): x \in X, y \in Y\}$, and $d(x, y)$ denotes the Euclidean distance between the points $x$ and $y$. The harmonicity of

$$
u_{\tau}=\operatorname{Im}\left(e^{-i \alpha} f_{\tau}\right)
$$

on $\mathbb{A}_{\rho}$ implies that the compositions $u_{\tau} \circ g_{k}$ are harmonic on $\mathbb{D}$ for all $k$, and thus

$$
\begin{aligned}
u_{\tau}\left(g_{k}\left(r_{k} e^{i t_{k}}\right)\right) & =\int_{-\pi}^{\pi} u_{\tau}\left(g_{k}\left(e^{i s}\right)\right) \cdot P_{k}\left(e^{i s}\right) \mathrm{d} s, \\
& =\left(\int_{-2 \delta}^{2 \delta}+\int_{J}\right) u_{\tau}\left(g_{k}\left(e^{i s}\right)\right) \cdot P_{k}\left(e^{i s}\right) \mathrm{d} s,
\end{aligned}
$$

where

$$
P_{k}\left(e^{i s}\right)=\frac{1-r_{k}^{2}}{2 \pi\left(1-2 r_{k} \cos \left(s-t_{k}\right)+r_{k}^{2}\right)}, \quad 0 \leq r_{k} \leq 1 .
$$

If $A_{k} \subseteq A$, then the first integral on the second line in (5.4) is non-negative by virtue of the fact that the restriction of $u_{\tau}$ to $A_{k}$ is non-negative. The second integral, on the other hand, may be estimated as follows. If $\varepsilon>0$, then on each set

$$
S_{k}=\left\{r_{k} e^{i t_{k}} \in \mathbb{D}: 1-\frac{\varepsilon \sin ^{2} \delta}{4\left|a^{\prime}\right|}<r_{k} \leq 1,-\delta<t_{k}<\delta\right\},
$$

we have

$$
\left|\int_{J} u_{\tau}\left(g_{k}\left(e^{i s}\right)\right) \cdot P_{k}\left(e^{i s}\right) \mathrm{d} s\right| \leq \frac{2\left|a^{\prime}\right|\left(1-r_{k}^{2}\right)}{\sin ^{2} \delta}<\frac{4\left|a^{\prime}\right|\left(1-r_{k}\right)}{\sin ^{2} \delta}<\varepsilon .
$$

Hence, for each $\varepsilon>0$, there is a set $S_{k}$ containing $g_{k}^{-1}\left(A_{k}\right)$ such that

$$
\left(u_{\tau} \circ g_{k}\right)>-\varepsilon .
$$

A similar argument applied to each of the other cases

$$
A_{k} \subseteq B, A_{k} \subseteq C, A_{k} \subseteq D
$$

(with $\alpha$ replaced by $\beta, \alpha+\pi, \beta+\pi$, respectively) also yields $S_{k} \supset g_{k}^{-1}\left(A_{k}\right)$ such that $(5.5)$ is valid. 
Fix $r$ sufficiently close to 1 such that

$$
\mathbb{T}_{r} \subset \bigcup_{k=1}^{n} g_{k}\left(S_{k}\right) \backslash \mathbb{T} .
$$

Since $f_{\tau}$ is non-zero on the compact set $\mathbb{T}_{r}$, there exists $m_{r}>0$ such that

$$
\left|f_{\tau}\right| \geq m_{r}
$$

on $\mathbb{T}_{r}$. Pick $k_{r}>0$ satisfying

$$
\varepsilon_{r}=\arcsin \frac{k_{r}}{m_{r}}<\frac{1}{2} \min \{\beta-\alpha, \alpha+\pi-\beta\} .
$$

Since

$$
u_{\tau}=\operatorname{Im}\left(e^{-i \alpha} f_{\tau}\right)=\left|f_{\tau}\right| \sin \left(\psi_{\tau}-\alpha\right),
$$

it follows from (5.7) that on $g_{k}\left(S_{k}\right) \cap \mathbb{T}_{r}$,

$$
\sin \left(\psi_{\tau}-\alpha\right)>-\frac{k_{r}}{m_{r}}
$$

if $A_{k} \subseteq A$, and thus

$$
-\varepsilon_{r}<\psi_{\tau}-\alpha<\pi+\varepsilon_{r} .
$$

Likewise, on $g_{k}\left(S_{k}\right) \cap \mathbb{T}_{r}$,

$$
\begin{cases}-\varepsilon_{r}<\psi_{\tau}-\beta<\pi+\varepsilon_{r} & \text { if } A_{k} \subseteq B ; \\ -\varepsilon_{r}<\psi_{\tau}-(\alpha+\pi)<\pi+\varepsilon_{r} & \text { if } A_{k} \subseteq C ; \\ -\varepsilon_{r}<\psi_{\tau}-(\beta+\pi)<\pi+\varepsilon_{r} & \text { if } A_{k} \subseteq D .\end{cases}
$$

In particular, on $\mathbb{T}_{r}$, we obtain

$$
\psi_{\tau}\left(r e^{i(\theta+2 \pi)}\right)=\psi_{\tau}\left(r e^{i \theta}\right)+2 \pi,
$$

though this may also be seen from the fact that $h\left(\mathbb{T}_{r}\right)$ is a Jordan curve. Hence, $\psi_{\tau}-\theta$ is a single-valued harmonic function on $\mathbb{A}_{\rho}$.

In view of (5.8), (5.9), and (5.10), we see that $\psi_{\tau}\left(r e^{i \theta}\right)-\theta$ is uniformly bounded for all $\tau$ and $r$ satisfying (5.2) and (5.6), respectively. A similar argument could be applied to $\mathbb{T}_{\rho}$ to obtain a corresponding result when $r$ is sufficiently close to $\rho$. This proves Lemma 5.1, since $\psi_{\tau}-\theta$ is continuous on $\mathbb{A}_{\rho}$. 


\section{Proof of Theorem 2.2}

Let $\psi(z)=\arg (\partial / \partial \theta) h(z)$ for all $z=r e^{i \theta} \in \mathbb{A}_{\rho}$. As a consequence of (5.1), the convexity of $h\left(\mathbb{T}_{r}\right)$ will follow from the inequality

$$
\frac{\partial \psi}{\partial \theta} \geq 0
$$

on $\mathbb{A}_{\rho}$, with $h\left(\mathbb{T}_{r}\right)$ being strictly convex if the inequality is strict. Since $h\left(\mathbb{T}_{r}\right)$ is a smooth (or, more precisely, real-analytic) Jordan curve, we obtain

$$
\psi\left(r e^{i(\theta+2 \pi)}\right)=\psi\left(r e^{i \theta}\right)+2 \pi,
$$

Observe that

$$
\frac{\partial h}{\partial \theta}=i\left(z h_{z}-\bar{z} h_{\bar{z}}\right)=i z h_{z}\left(1-\frac{\bar{z} h_{\bar{z}}}{z h_{z}}\right)
$$

By (3.2), the quantity in parentheses has positive real part and hence, by (2.1), its argument is a single-valued harmonic function. Since $h_{z}$ is holomorphic and non-zero on $\mathbb{A}_{\rho}$, it follows from (6.2) and (6.3) that $\psi-\theta$ is a single-valued harmonic function on $\mathbb{A}_{\rho}$. Moreover, it is bounded by virtue of Lemma 5.1 since $\psi=\lim _{\tau \rightarrow 0} \psi_{\tau}$ on $\mathbb{A}_{\rho}$.

Let $G_{z}(\zeta)$ denote the Green's function for $\mathbb{A}_{\rho}$ with singularity at $z=r e^{i \theta}$, and let $n=n_{w}$ be the inward normal at $w=R e^{i \varphi} \in \partial \mathbb{A}_{\rho}$. We may rotate $\mathbb{A}_{\rho}$ together with the singularity $z=r e^{i \theta}$ about the origin through an angle $\sigma$ to obtain

$$
G_{r e^{i \theta}}\left(R e^{i \varphi}\right)=G_{r e^{i(\theta+\sigma)}}\left(R e^{i(\varphi+\sigma)}\right),
$$

from which the definition of partial differentiation implies

$$
\begin{aligned}
\frac{\partial}{\partial \theta} G_{z}(w) & =\lim _{\sigma \rightarrow 0} \frac{1}{\sigma}\left(G_{r e^{i(\theta+\sigma)}}\left(R e^{i \varphi}\right)-G_{r e^{i \theta}}\left(R e^{i \varphi}\right)\right) \\
& =\lim _{\sigma \rightarrow 0} \frac{1}{\sigma}\left(G_{r e^{i \theta}}\left(R e^{i(\varphi-\sigma)}\right)-G_{r e^{i \theta}}\left(R e^{i \varphi}\right)\right) \\
& =\lim _{\sigma \rightarrow 0} \frac{1}{-\sigma}\left(G_{r e^{i \theta}}\left(R e^{i(\varphi+\sigma)}\right)-G_{r e^{i \theta}}\left(R e^{i \varphi}\right)\right) \\
& =-\frac{\partial}{\partial \varphi} G_{z}(w) .
\end{aligned}
$$

Hence,

$$
\frac{\partial}{\partial \theta} \frac{\partial}{\partial n} G_{z}(w)=\frac{\partial}{\partial n} \frac{\partial}{\partial \theta} G_{z}(w)=-\frac{\partial}{\partial n} \frac{\partial}{\partial \varphi} G_{z}(w)=-\frac{\partial}{\partial \varphi} \frac{\partial}{\partial n} G_{z}(w)
$$


Let $T=\left\{t \in \mathbb{R}:(\partial / \partial t) \Psi_{1}(t)\right.$ and $(\partial / \partial t) \Psi_{\rho}(t)$ both exist $\}$, where

$$
\Psi_{1}(\theta)=\arg \frac{\partial}{\partial \theta} h\left(e^{i \theta}\right), \quad \Psi_{\rho}(\theta)=\arg \frac{\partial}{\partial \theta} h\left(\rho e^{i \theta}\right) .
$$

Since the boundary components of $h\left(\mathbb{A}_{\rho}\right)$ are convex Jordan curves by Corollary 4.2 , it follows that $\mathbb{R} \backslash T$ is countable. Recall that the harmonic function $\psi-\theta$ has the integral representation (see, e.g., [17, Theorem I.21])

$$
\begin{aligned}
2 \pi(\psi(z)-\theta)=\int_{0}^{2 \pi} & {\left[\Psi_{1}(\varphi)-\varphi\right] \frac{\partial}{\partial n} G_{z}\left(e^{i \varphi}\right) \mathrm{d} \varphi } \\
& +\int_{0}^{2 \pi}\left[\Psi_{\rho}(\varphi)-\varphi\right] \frac{\partial}{\partial n} G_{z}\left(\rho e^{i \varphi}\right) \rho \mathrm{d} \varphi,
\end{aligned}
$$

where the integrals are taken over $[0,2 \pi] \cap T$. Partial differentiation with respect to $\theta$ followed by an application of (6.5) yields

$$
\begin{aligned}
& 2 \pi\left(\frac{\partial}{\partial \theta} \psi(z)-1\right) \\
= & \int_{0}^{2 \pi}\left[\Psi_{1}(\varphi)-\varphi\right] \frac{\partial}{\partial \theta} \frac{\partial G_{z}}{\partial n} \mathrm{~d} \varphi+\int_{0}^{2 \pi}\left[\Psi_{\rho}(\varphi)-\varphi\right] \frac{\partial}{\partial \theta} \frac{\partial G_{z}}{\partial n} \rho \mathrm{d} \varphi \\
= & -\int_{0}^{2 \pi}\left[\Psi_{1}(\varphi)-\varphi\right] \frac{\partial}{\partial \varphi} \frac{\partial G_{z}}{\partial n} \mathrm{~d} \varphi-\int_{0}^{2 \pi}\left[\Psi_{\rho}(\varphi)-\varphi\right] \frac{\partial}{\partial \varphi} \frac{\partial G_{z}}{\partial n} \rho \mathrm{d} \varphi \quad \text { by }(6.5) \\
= & \int_{0}^{2 \pi} \frac{\partial G_{z}}{\partial n} \mathrm{~d}\left[\Psi_{1}(\varphi)-\varphi\right]+\int_{0}^{2 \pi} \frac{\partial G_{z}}{\partial n} \rho \mathrm{d}\left[\Psi_{\rho}(\varphi)-\varphi\right] .
\end{aligned}
$$

Hence (see, e.g., [17, Theorem I.20]),

$$
2 \pi \frac{\partial}{\partial \theta} \psi(z)=\int_{0}^{2 \pi} \frac{\partial G_{z}}{\partial n} \mathrm{~d} \Psi_{1}(\varphi)+\int_{0}^{2 \pi} \frac{\partial G_{z}}{\partial n} \rho \mathrm{d} \Psi_{\rho}(\varphi) .
$$

It follows from (5.1) that $\Psi_{1}(\varphi)$ and $\Psi_{\rho}(\varphi)$ are non-decreasing functions of $\varphi$. Since $\partial G / \partial n$ is positive on $\partial \mathbb{A}_{\rho}$, it follows from (6.6) that $\partial \psi / \partial \theta$ is also positive on $\mathbb{A}_{\rho}$. Hence, $h\left(\mathbb{T}_{r}\right)$ is strictly convex for $\rho<r<1$, which concludes our proof of Theorem 2.2.

Remark. The proof would have been much simpler if $h \in C^{2}\left(\overline{\mathbb{A}_{\rho}}\right)$, for it follows from (6.3) that (6.1) is then equivalent to

$$
\begin{aligned}
1+ & \frac{\partial}{\partial \theta}\left\{\arg h_{z}+\arg \left(1-\frac{\bar{z} h_{\bar{z}}}{z h_{z}}\right)\right\} \\
& =1+\operatorname{Re}\left\{\frac{z h_{z z}}{h_{z}}+\frac{\bar{z}\left(z h_{\bar{z}} h_{z z}+2 h_{z} h_{\bar{z}}+\bar{z} h_{z} h_{\bar{z} \bar{z}}\right)}{h_{z}\left(z h_{z}-\bar{z} h_{\bar{z}}\right)}\right\} \geq 0 .
\end{aligned}
$$

Since this holds on $\partial \mathbb{A}_{\rho}$, the maximum principle yields the same inequality on $\mathbb{A}_{\rho}$. The desired conclusion then follows from the observation that $\partial \psi / \partial \theta$ cannot be identically zero on $\mathbb{A}_{\rho}$, as $h\left(\mathbb{T}_{r}\right)$ is a Jordan curve for $\rho<r<1$. 
Acknowledgements. The author thanks Leonid Kovalev for valuable discussions on the subject. The author also thanks the referee for carefully reading several versions of the manuscript.

\section{REFERENCES}

[1] K. Astala, T. IWANIEC, and G. Martin, Deformations of annuli with smallest mean distortion, Arch. Ration. Mech. Anal. 195 (2010), no. 3, 899-921. http://dx.doi.org/10.1007/s00205-009-0231-z. MR2591976 (2011c:30059)

[2] J. Clunie and T. Sheil-SMall, Harmonic univalent functions, Ann. Acad. Sci. Fenn. Ser. A I Math. 9 (1984), 3-25. http://dx.doi.org/10.5186/aasfm.1984.0905. MR752388 (85i:30014)

[3] P. L. DuREN, Univalent functions, Grundlehren der Mathematischen Wissenschaften [Fundamental Principles of Mathematical Sciences], vol. 259, Springer-Verlag, New York, 1983. MR708494 (85j:30034)

[4] Harmonic Mappings in the Plane, Cambridge Tracts in Mathematics, vol. 156, Cambridge University Press, Cambridge, 2004.

http://dx.doi.org/10.1017/CBO9780511546600. MR2048384 (2005d:31001)

[5] A. W. GoOdman and E. B. SAFF, On univalent functions convex in one direction, Proc. Amer. Math. Soc. 73 (1979), no. 2, 183-187.

http://dx.doi.org/10.2307/2042288. MR516461 (80e:30006)

[6] E. HeinZ, On one-to-one harmonic mappings, Pacific J. Math. 9 (1959), no. 1, 101-105. http://dx.doi.org/10.2140/pjm.1959.9.101. MR0104933 (21 \#3683)

[7] W. HengarTNER and G. SCHOBER, A remark on level curves for domains convex in one direction, Applicable Anal. 3 (1973), no. 1, Collection of articles dedicated to Eberhard Hopf on the occasion of his 70 th birthday, 101-106.

http://dx.doi.org/1 0.1080/0003681 7308839059. MR0393450 (52 \#14260)

[8] Harmonic mappings with given dilatation, J. London Math. Soc. (2) 33 (1986), no. 3, 473-483. http://dx.doi.org/10.1112/jlms/s2-33.3.473. MR850963 (87j:30037)

[9] T. Iwaniec, N. T. KOH, L. V. KovaleV, and J. OnNinen, Existence of energy-minimal diffeomorphisms between doubly connected domains, Invent. Math. 186 (2011), no. 3, 667-707. http://dx.doi.org/10.1007/s00222-011-0327-6. MR2854087

[10] T. IWANIEC, L. V. KOVALEV, and J. ONNINEN, Hopf differentials and smoothing Sobolev homeomorphisms, Int. Math. Res. Not. IMRN 14 (2012), 3256-3277. http://dx.doi.org/10.1093/imrn/rnr144. MR2946225

[11] D. KALAJ, Energy-minimal diffeomorphisms between doubly connected Riemann surfaces, Calc. Var. Partial Differential Equations 51 (2014), no. 1-2, 465-494. http://dx.doi.org/10.1007/s00526-013-0683-8. MR3247397

[12] N. T. KOH and L. V. KOVALEV, Area contraction for harmonic automorphisms of the disk, Bull. Lond. Math. Soc. 43 (2011), no. 1, 91-96. http://dx.doi.org/10.1112/blms/bdq083. MR2765553 (2012a:31001)

[13] O. Lehto and K. I. Virtanen, Quasiconformal Mappings in the Plane, 2nd ed., Die Grundlehren der mathematischen Wissenschaften [Fundamental Principles of Mathematical Sciences], vol. 126, Springer-Verlag, New York-Heidelberg, 1973. Translated from the German by K.W. Lucas. MR0344463 (49 \#9202)

[14] H. LEWY, On the non-vanishing of the Jacobian in certain one-to-one mappings, Bull. Amer. Math. Soc. 42 (1936), no. 10, 689-692. http://dx.doi.org/10.1090/S0002-9904-1936-06397-4. MR1563404

[15] S. RUSCHEWEYH and L.C. SALinaS, On the preservation of direction-convexity and the Goodman-Saff conjecture, Ann. Acad. Sci. Fenn. Ser. A I Math. 14 (1989), no. 1, 63-73. http://dx.doi.org/1 0.5186/aasfm.1989.1427. MR997971 (90h:30041)

[16] E. STUDY, Vorlesungen ber ausgewhlte Gegenstnde der Geometrie, Teubner, Leipzig, 1913. Heft II. 
[17] M. Tsuj, Potential Theory in Modern Function Theory, Chelsea Publishing Co., New York, 1975. Reprinting of the 1959 original. MR0414898 (54 \#2990)

School of Mathematics and Statistics

University of Canterbury

Private Bag 4800

Christchurch 8140, New Zealand

E-MAIL: ngin-tee.koh@canterbury.ac.nz

KEY WORDS AND PHRASES: Annulus, convex, doubly-connected, energy-minimal diffeomorphisms, harmonic diffeomorphisms, harmonic homeomorphisms, harmonic mappings, hereditary property.

2010 Mathematics Subject Classification: 30C45, 30D40, 31A05, 31A20.

Received: February 4, 2013; revised: September 20, 2014. 